\title{
From orphan drugs to adopted therapies: Advancing C3-targeted intervention to the clinical stage
}

Dimitrios C. Mastellos ${ }^{1,2}$, Edimara S. Reis ${ }^{1}$, Despina Yancopoulou ${ }^{3}$, George Hajishengallis ${ }^{4}$, Daniel Ricklin $^{1}$ and John D. Lambris ${ }^{1}$

\footnotetext{
${ }^{1}$ Department of Pathology and Laboratory Medicine, Perelman School of Medicine, University of Pennsylvania, Philadelphia PA 19104, USA; ${ }^{2}$ Division of Biodiagnostic Sciences and Technologies, INRASTES, National Center for Scientific Research 'Demokritos', Aghia Paraskevi Attikis, 15310 Athens, Greece; ${ }^{3}$ Amyndas Pharmaceuticals, Glyfada, 16675 Athens, Greece; ${ }^{4}$ Department of Microbiology, School of Dental Medicine, University of Pennsylvania, Philadelphia, PA 19104, USA.
}

Running title: C3 therapeutics in clinical development

\section{Corresponding author:}

John D. Lambris, Ph.D.

Perelman School of Medicine

University of Pennsylvania

401 Stellar Chance Laboratories

422 Curie Blvd

Philadelphia, PA 19104

USA

Tel.: +1 215-746-5765

E-mail: lambris@upenn.edu 


\begin{abstract}
Complement dysregulation is increasingly recognized as an important pathogenic driver in a number of clinical disorders. Complement-triggered pathways intertwine with key inflammatory and tissue destructive processes that can either increase the risk of disease or exacerbate pathology in acute or chronic conditions. The launch of the first complement-targeted drugs in the clinic has undeniably stirred the field of complement therapeutic design, providing new insights into complement's contribution to disease pathogenesis and also helping to leverage a more personalized, comprehensive approach to patient management. In this regard, a rapidly expanding toolbox of complement therapeutics is being developed to address unmet clinical needs in several immune-mediated and inflammatory diseases. Elegant approaches employing both surface-directed and fluid-phase inhibitors have exploited diverse components of the complement cascade as putative points of therapeutic intervention. Targeting C3, the central hub of the system, has proven to be a promising strategy for developing biologics as well as small-molecule inhibitors with clinical potential. Complement modulation at the level of C3 has recently shown promise in preclinical primate models, opening up new avenues for therapeutic intervention in both acute and chronic indications fueled by uncontrolled C3 turnover. This review highlights recent developments in the field of complement therapeutics, focusing on C3-directed inhibitors and alternative pathway (AP) regulator-based approaches. Translational perspectives and considerations are discussed, particularly with regard to the structure-guided drug optimization and clinical advancement of a new generation of C3-targeted peptidic inhibitors.
\end{abstract}

\title{
Keywords:
}

Complement dysregulation, C3 convertase, AMY-101, clinical efficacy, primate models, peptidic C3 inhibitors 


\section{Introduction}

\section{Complement-targeted drug discovery: a changing landscape with emerging opportunities}

Traditionally perceived as a phylogenetically conserved antimicrobial sentinel with enhanced pathogenneutralizing and 'waste disposal' capabilities, complement had remained for several decades behind the curtains of mainstream immunological research. However, the advent of high-throughput gene profiling and other technological advancements has led to unprecedented discoveries in preclinical models and large-scale patient cohorts that implicate complement dysregulation in the pathogenesis of several immune-mediated and inflammatory diseases (Ricklin and Lambris, 2013a). It is now widely appreciated that complement plays a multitasking role in tissue immunosurveillance and homeostasis by extending vital links to diverse immunoregulatory networks (Ricklin et al., 2010;Kolev et al., 2014). While tightly regulated under steady-state conditions, this innate immune system is vulnerable to subtle genetic and epigen acquired (e.g. autoimmune-driven) alterations of its activity that can collectively tilt the balance of tissue immunosurveillance toward a persistent host-directed attack, with detrimental proinflammatory consequences (Ricklin and Lambris, 2013a). Such regulatory perturbations can skew clinical phenotypes, either by increasing the risk for a disease or exacerbating the clinical manifestations of a chronic condition.

Seminal studies employing genome-wide association studies (GWAS) and next-generation sequencing have provided important insights into complement's involvement in human pathophysiology by revealing that subtle functional modifications of key complement activities (via mutations or common polymorphisms) can unleash a derailed complement response on host surfaces that culminates in inflammatory tissue damage and chronic pathology (Schramm et al., 2014;Ricklin and Lambris, 2013a). The predisposing role of certain complement gene polymorphisms in age-related macular degeneration (AMD) is an illustrative example of how this complex interplay can influence disease susceptibility (Schramm et al., 2014). Clearly, complement dysregulation has been recognized as a key pathogenic driver in certain rare but chronic debilitating diseases, including paroxysmal nocturnal hemoglobinuria (PNH) and atypical hemolytic uremic syndrome (aHUS) (Reis et al., 2015). In fact, therapeutic intervention in these diseases using complement inhibitors has led to remarkable clinical benefit, restoring complement imbalance and drastically improving therapeutic outcomes in patients. The expanding spectrum of complement-mediated clinical disorders, together with the successful bedside application of the first complement-specific drugs and the clinical experience gained thus far have 
vigorously rekindled the interest of pharmaceutical companies in the development and clinical advancement of new complement-targeted drug leads (Morgan and Harris, 2015;Reis et al., 2015).

Nevertheless, the path to clinical intervention has remained challenging, particularly in light of recent findings that argue for a more complex impact of the "complotype" on disease pathogenesis and therapeutic responses (Harris et al., 2012). For instance, a fair proportion of PNH patients receiving treatment with first-in-class anti-complement agents (i.e., eculizumab) have shown an insufficient response to this standard anti-C5 therapy (Risitano, 2013;Nishimura et al., 2014). These unmet clinical needs have shed light on previously elusive pathogenetic mechanisms that should guide alternative therapeutic interventions. C3 opsonin-mediated extravascular hemolysis in PNH patients under eculizumab treatment is an emerging clinical observation that calls for the evaluation of new treatment options (Mastellos et al., 2014). Therapy-modifying effects have also been ascribed to genetic alterations affecting complement genes (e.g., C5 and CR1) in certain PNH patients (Rondelli et al., 2014;Nishimura et al., 2014). The growing appreciation that a fraction of patients are refractory to standard therapeutic regimens because of genetic variance, pharmacologic breakthrough phenomena, or ongoing opsonization has prompted the design of new complement-targeted therapeutic modalities (e.g., C3 therapeutics) that can effectively circumvent these limitations, consolidating potential variations in patient responses. Of note, the emerging recognition of complement's involvement in conditions that still remain orphan despite the availability of options in the clinic (e.g., C3 glomerulopathy) further underscores the need for alternative therapeutic strategies.

Overall, genomic and epidemiological studies and clinical follow-up observations in patients undergoing complement intervention have reshaped our choice of overarching tenets to guide complement therapeutic design in the future. Whatever the favored approach or target protein, the ultimate goal should be to design a more comprehensive therapeutic protocol targeting the desired complement activity in the clinic (i.e., through a more personalized medical approach). While acknowledging the remarkable versatility and ingenuity of the various "schools" of complement therapeutic design, we have focused this review on emerging trends and opportunities regarding the translational potential of next-generation C3-targeted therapeutics.

\section{Current trends in complement-targeted therapeutic intervention}

Complement offers ample opportunity for targeted therapeutic modulation, at various steps of the cascade and in a disease-specific context. Although only two complement-targeted drugs have thus far 
entered the clinic, the therapeutic anti-C5 antibody eculizumab (Soliris; Alexion Pharmaceuticals) and various preparations of the physiological regulator C1 esterase inhibitor (C1-INH), several new candidate drugs targeting various components of the cascade are currently in different stages of clinical development (Ricklin and Lambris, 2013b;Morgan and Harris, 2015; Reis et al., 2015). In terms of strict complement specificity, it should be emphasized that while C1-INH effectively blocks complement activation via the classical or lectin pathways, it also exerts broader modulation over various bloodborne proteolytic cascades, including the coagulation and kallikrein/bradykinin pathways. Therefore its therapeutic impact in complement-driven pathologies has to be considered under a broader inhibitory scope.

Being an intricate protein-protein interaction network that is finely regulated by fluid-phase and surfacebound regulators, complement taps into several immunoregulatory and proinflammatory pathways recruiting numerous pattern-recognition molecules, protein assemblies, cofactors, and receptors (Ricklin et al., 2010). Complement activation can proceed via any of three canonical pathways (alternative, lectin, classical) that converge at the cleavage of the third complement component (C3) through sequential proteolytic steps (Figure 1). Large multi-protein enzymatic complexes, termed convertases, are responsible for the proteolytic activation of the central components $\mathrm{C} 3$ and $\mathrm{C} 5$ and the subsequent release of their respective bioactive fragments: C3a and C3b, C5a and C5b, respectively. Regardless of the initial route of activation, amplification of C3b deposition via the alternative pathway (AP) may lead to high opsonic turnover on target surfaces and thus has been implicated as a key pathogenic mechanism underlying several diseases. In fact, AP-mediated amplification of complement responses has been shown to account for more than $80 \%$ of the downstream activation that is elicited following initial triggering of the CP or LP in certain pathophysiological settings (Harboe et al., 2004; Harboe et al., 2009). C5b forms the initial component of the terminal (lytic) pathway, promoting a multi-protein assemblage that leads to the formation of the cell-perforating membrane attack complex (MAC, C5b-9) (Ricklin and Lambris, 2013a). The direct convertase-independent cleavage of C3 and C5, mainly by extrinsic proteases of the coagulation and fibrinolytic systems, has also been reported under certain pathophysiological circumstances (Huber-Lang et al., 2006).

Blockade of a specific initiation route (AP, lectin, or classical) offers a targeted therapeutic approach that blunts upstream complement activation while sparing the immunomodulatory and homeostatic functions of downstream complement effectors/pathways. However, this approach might have only minimal therapeutic merit if multiple initiation routes converge in triggering pathological 
sequelae in the clinical condition in question. There are, however, disorders that might benefit from targeting a single pathway, including autoimmune hemolytic anemias, since complement-mediated hemolysis in these conditions is mainly triggered by the classical pathway (CP) via binding of autoreactive IgGs and IgMs to circulating erythrocytes (Berentsen, 2015). Although preclinical evidence from animal models points to a distinct role of the LP in triggering pathology in certain diseases, the clinical impact of specifically targeting LP activation still needs to be further explored.

From a different standpoint, strategies targeting the central protein, C3, offer a broader and more comprehensive interception by blunting amplification and downstream receptor-triggered effector mechanisms, or even all activation routes, as exemplified by compstatin-based C3 inhibitors and engineered versions of natural C3 regulators (Ricklin and Lambris, 2016b). Such a broadly inhibiting approach may have greater therapeutic leverage in clinical conditions associated with systemic complement activation. In general, any complement-targeted therapeutic protocol will require a thorough consideration of potential prophylactic measures that can compensate for a likely compromised response in terms of microbial immune surveillance, particularly in chronic interventions (Ricklin and Lambris, 2016a).

Targeting the terminal pathway may prove rewarding in clinical conditions that are largely driven by terminal pathway effectors such as $\mathrm{C} 5 \mathrm{a}$ and the MAC. Blockade of the terminal pathway via C5 targeting abrogates the detrimental proinflammatory consequences of the C5a-C5aR axis and simultaneously affords protection from the cytolytic effects of the MAC on autologous surfaces, while also preserving upstream homeostatic responses driven by C3 activation and AP amplification. Alternatively, specific interception of C5aR1 signaling (e.g., using small-molecule antagonists) appears to be a more tailored approach for conditions largely fueled by C5a-mediated inflammatory activation, because this approach preserves the bactericidal activity of complement (in serum) elicited by MAC assembly on complement-sensitive microbial surfaces (Sprong et al., 2003; Ricklin and Lambris, 2013c). Given that $\mathrm{C} 5 \mathrm{a}$ can trigger divergent cellular responses by binding to two receptors (i.e., C5aR1/CD88 and C5aR2/C5L2) with distinct and perhaps even opposing functions, therapeutic targeting of C5a (e.g. via antibody-based blockade) should be distinguished from the pharmacological antagonism of any of the two C5a receptors. Selection of the optimal target or pathway for intervention inextricably depends on its disease-specific contribution and the underlying mechanisms driving pathology in that particular clinical condition.

Fortunately, the complement drug discovery field has witnessed a surge in the development of diversified and mechanistically subtle approaches for inhibiting complement at various steps of the 
cascade. Potent and highly selective inhibitors have been registered in the pipeline of pharmaceutical companies in an all-inclusive scope ranging from small-sized molecules to larger biologics (Morgan and Harris, 2015). Since most of the activities of complement rely on protein-protein interactions (PPI), the development of biologics (e.g., therapeutic antibodies) has gained considerable traction for blocking larger contacts and interfaces (exosites) uniquely involved in substrate recognition and binding. An illustrative example of the Ab-based approach is a humanized anti-FD Fab (lampalizumab, Genentech/Roche) that specifically targets the FD exosite to prevent activation of the AP C3 proconvertase (C3bB) (Katschke, Jr. et al., 2012). This antibody blocks AP activation and has shown clinical potential for the treatment of geographic atrophy (GA), an advanced dry form of age-related macular degeneration (AMD) (Regillo C.D. et al., 2013), after local administration. Other elegant approaches exploiting biologics as potential PPI inhibitors include recombinant fusion proteins encompassing regulatory (CCP) modules of natural regulators (e.g., TT30, miniFH) (Fridkis-Hareli et al., 2011;Schmidt et al., 2013), parasite-secreted immune evasion proteins (e.g., OmCl; (Roversi et al., 2007), recombinant protein scaffolds (e.g. SOBI002) (Swedish Orphan Biovitrum, 2016), and oligonucleotide-based ligands such as aptamers (e.g., Zimura), liver-targeted RNAi therapeutics (ALN-CC5, Alnylam), or Spiegelmers (e.g., NOX-D21) (Ricklin and Lambris, 2016b). The development of highly potent peptidic inhibitors has defined a unique class of small-sized complement therapeutics that have shown great promise for clinical translation in various indications. This approach has yielded specific C5aR1 antagonists (e.g., PMX53) (Woodruff et al., 2011), potent C3-targeted peptide inhibitors of the compstatin family (e.g., the Cp40-based therapeutic AMY-101, Amyndas Pharmaceuticals)(Mastellos et al., 2015), FH-binding peptides with promising results in models of biomaterial-triggered thromboinflammation (Wu et al., 2011) (Ricklin and Lambris, 2016a), and a peptide-based C5 inhibitor (Ra Pharmaceuticals) (Ra Pharmaceuticals, 2016). As compared to larger biologics, these peptide-based therapeutics are expected to offer advantages regarding lowered production costs and better tissue distribution; however, their clinical efficacy will need to be closely monitored during clinical development.

\section{Complement interception: systemic vs. locally targeted}

Complement-based therapeutic design has been directly influenced by concepts and approaches originating from two intersecting schools of thought: i) the "systemic interception" approach, which targets complement proteins in the fluid phase using biologics or small-sized inhibitors, and ii) the "surface-targeting" approach, which directs natural or engineered chimeric inhibitors closer to the complement-opsonized surface [reviewed in (Ricklin and Lambris, 2013c)]. In general terms, systemic 
approaches may better apply to conditions in which complement dysregulation impacts multiple physiological systems, leading to adverse systemic inflammatory responses that propagate to vital target organs. In contrast, targeting complement inhibitors to the diseased or incompatible xenogeneic surface is considered advantageous when the pathological ramifications are mainly driven by a local complement imbalance (e.g., a lack of surface-bound C3 regulators), rendering a host surface prone to uncontrollable complement attack. This surface-targeted approach may also be beneficial in conditions in which persistent C3 opsonic turnover fuels a vicious cycle of inflammation on malignantly transformed surfaces (e.g., tumor cells), transplants, or biomaterials (Ekdahl et al., 2011). Indeed, promising examples of this surface-directed therapeutic approach include fusion proteins (e.g., TT30 [FH-CR2]), condensed versions of AP regulators with enhanced surface recognition and inhibitory potency (e.g., mini-FH), and targeting moieties that recruit soluble regulators to biomaterial surfaces, thereby attenuating AP activation and C3 opsonization (e.g., FH-binding peptides) [reviewed in (Ricklin and Lambris, 2016b)]. New insights into the unique surface selectivity of this new class of C3-targeted inhibitors and the therapeutic prospects emerging from their preclinical evaluation are discussed below.

\section{Therapeutic intervention at the level of $\mathrm{C3}$}

Positioned at the intersection of all complement activation pathways, C3 is a highly attractive target for developing complement therapeutics with broad inhibitory potency. All initiation routes, regardless of their trigger, lead to the formation of C3 convertases that cleave native C3 into C3b and C3a (Ricklin et al., 2010) (Figure 1). C3a is generally considered a potent proinflammatory mediator that can activate a multitude of myeloid or non-myeloid cells through binding to its G-protein-coupled C3aR receptor. Conversely, recent evidence has suggested that this C3-derived peptide may also exert antiinflammatory activity in certain pathophysiological contexts (Wu et al., 2013; Coulthard and Woodruff, 2015). Meanwhile, the C3b opsonin rapidly decorates surfaces undergoing complement attack and forms the initial component of the AP C3 convertase (C3bBb). Increased C3b deposition via the CP, or the AP amplification loop is both a prerequisite for the generation of C5 convertases, which cleave C5 into $\mathrm{C} 5 \mathrm{~b}$ and the proinflammatory mediator $\mathrm{C} 5 \mathrm{a}$, and also a common denominator exacerbating the clinical course of several complement-driven pathologies. Irrespective of the initial route of activation, AP amplification and aberrant C3b deposition on host surfaces or biomaterials have been implicated as a pivotal pathogenic driver fueling the tissue-destructive, vicious cycle of inflammation in several conditions (Ricklin and Lambris, 2013a).Notably, the presence of a contingent of fluid-phase or surface- 
bound C3 regulators that can dismantle C3 convertases or promote the factor I (FI)-dependent degradation of $\mathrm{C} 3 \mathrm{~b}$ ensures the containment of complement responses on host surfaces. Nevertheless, subtle genetic or functional alterations can tilt the balance between C3 activation and C3b degradation toward a cell-injurious phenotype that ultimately exacerbates the course of the disease (Ricklin and Lambris, 2016a).

Despite the context- and disease-specific contribution of each complement pathway (i.e., upstream or downstream of (3), C3 interception appears to be a more comprehensive strategy for blocking complement activation because it also abrogates the pathological consequences of aberrant AP amplification and terminal pathway activation (i.e., MAC-mediated cytolysis and C5a-triggered responses). It is increasingly recognized that C3-targeted therapeutics hold promise for the treatment of disorders largely fueled by AP amplification and uncontrollable C3 deposition on host surfaces, such as C3 glomerulopathies (C3G) (Smith et al., 2007), and for disorders with broader complement involvement, such as AMD (Schramm et al., 2014). Furthermore, C3 inhibitors offer alternative therapeutic avenues that might confer even greater clinical benefit for patients who are already receiving standard anti-complement therapy, as in the case of individuals with $\mathrm{PNH}$.

C3 is a large and abundant plasma protein that undergoes a series of conformational transitions during its activation, releasing bioactive fragments that engage in numerous protein-protein interactions (Gros et al., 2008). Its dynamic conformational rearrangement exposes large surfaces for binding and modulation of its activity by biologics or small molecules that may act as protein-protein interaction (PPI) inhibitors. To date, several approaches have yielded both larger biologics and also small-molecule inhibitors of C3 activation, which act on native C3 itself, on the C3 convertase, or on the C3b breakdown cycle (Ricklin and Lambris, 2016a). Targeting native C3 with therapeutic antibodies remains challenging, but significant progress toward clinical translation has been made with small-sized peptidic inhibitors directly binding to native C3 (Mastellos et al., 2015). While earlier analogs of these C3-binding peptides have entered clinical development for different indications, a new series of these C3-targeted inhibitors has emerged with increased potency, favorable pharmacokinetic profiles, and sustained therapeutic efficacy in primate models that make them promising candidates for clinical intervention.

\subsection{Aiming at the core: $\mathrm{C} 3$-targeted peptidic inhibitors}


In contrast to developing therapeutic antibodies that bind C5 to inhibit its convertase-dependent activation (e.g., eculizumab), developing C3 therapeutics that act directly on native C3 to block its cleavage by C3 convertases has proven quite a challenging trail. To date, the only class of C3 inhibitors that binds directly to native $\mathrm{C} 3$, showing sustained inhibitory potency in preclinical models and potential for complement modulation in a number of clinical indications, is the compstatin family of peptidic C3 inhibitors (Ricklin and Lambris, 2008;Mastellos et al., 2015). Indeed, both early and next-generation compstatin analogs are now advancing through clinical development for indications ranging from dry $\mathrm{AMD}$ and $\mathrm{PNH}$ to $\mathrm{ABO}$-incompatible kidney transplantation, periodontal disease, and C3G (Ricklin and Lambris, 2015).

Compstatin was discovered through a phage-display library screening as a 13-residue cyclic peptide that selectively binds to native C3 and its bioactive fragments C3b/iC3b/C3c (Sahu et al., 1996). This C3-binding peptide exerts its broad and potent inhibitory function by blocking C3 activation by all convertases, thus preventing C3b-opsonization and blunting essentially all initiation, amplification, and downstream effector responses (Sahu et al., 2000;Ricklin and Lambris, 2008). Resolution of a co-crystal structure between an early compstatin analog and human C3c (Janssen et al., 2007) revealed important structural features that have guided subsequent optimization efforts and propelled our understanding of its unique binding and inhibitory mode on C3. By binding to a shallow pocket in the $\beta$-chain of C3, compstatin acts as a PPI inhibitor, sterically hindering the binding and cleavage of native C3 by C3 convertases (Janssen et al., 2007; Ricklin and Lambris, 2008). However, it is important to note that compstatin and its latest analogs neither prevent the direct cleavage of C3 by coagulation proteases nor the spontaneous hydrolysis of $\mathrm{C} 3$ into $\mathrm{C} 3\left(\mathrm{H}_{2} \mathrm{O}\right)$ (i.e., C3 "tick-over"). Moreover, C4b opsonization via CP/LP activation remains intact in the presence of compstatin (Mastellos et al., 2015).

Resolution of the structure of compstatin in its bound conformation has facilitated rigorous structure-function optimization of the parental peptide, resulting in improved compstatin derivatives with enhanced inhibitory potency, prolonged plasma stability, and target binding affinity almost 6000fold greater than the original peptide (reviewed in (Qu et al., 2009;Ricklin and Lambris, 2008;Mastellos et al., 2015)). A combination of enthalpy-driven peptide modifications (i.e. backbone N-methylation) that approximated the C3-bound structure of compstatin and increased hydrophobic interactions with the binding pocket in C3, along with C-terminal substitutions and a structure-guided extension of the $\mathrm{N}$ terminus with non-proteinogenic amino acids (e.g., D-Tyr) likely affording additional contacts with C3, have led to a remarkable increase in compstatin's inhibitory potency and binding affinity (Qu et al., 2013). Collectively, these peptide modifications built the basis for the development of the potent 
derivatives Cp20 and Cp40, which exhibit low or sub-nanomolar affinity for C3, and the Cp40-based therapeutic AMY-101, which is currently under clinical development by Amyndas Pharmaceuticals (Mastellos et al., 2015).

Early in the preclinical development phase, it became evident that compstatin-based inhibitors exhibit narrow species specificity, binding exclusively to human and non-human primate (NHP) C3 (Sahu et al., 2003). This specificity for primate C3 was largely explained when the structure of the binding site of compstatin on human C3 was released (Janssen et al., 2007). While critical residues of the MG4 and MG5 domains of the $\beta$-chain, which mediate the interaction with compstatin, remain conserved in human and primate C3, these exact residues vary in other mammalian species (Janssen et al, 2007), predicting subtle structural perturbations that likely obstruct the access of compstatin to its binding site on C3 molecules of non-primate origin.

A first milestone in compstatin's preclinical development was reached by the $4(1 \mathrm{MeW})$ analog, which displayed sustained inhibitory potency and therapeutic efficacy in NHP models of systemic inflammatory response syndrome (bacterial sepsis) (Silasi-Mansat et al., 2010)) and early-onset AMD (Chi et al., 2010). These studies underscored the clinical potential of this analog, propelling the clinical evaluation of a 4(1MeW)-based candidate (POT-4; Potentia Pharmaceuticals, now Apellis) as a therapeutic option for local (i.e., intravitreal) C3 interception in AMD in phase I and II trials (Alcon Research, 2013). In the meantime, different formulations of the same analog (APL-1 and its PEGylated derivative, APL-2) are being clinically developed by Apellis for both local and systemic applications ranging from $A M D$ and $P N H$ to COPD and asthma.

As opposed to the prolonged efficacy afforded by local application of compstatin analogs in AMD or periodontitis (discussed below), systemic administration of peptidic C3 inhibitors presents challenges with regard to pharmacokinetics and plasma stability. In this regard, extended pharmacokinetic analysis of next-generation compstatin analogs (e.g., Cp40) in NHP models has revealed a target-driven, biphasic plasma elimination profile that is marked by a rapid initial clearance, followed by a slower second phase of elimination that is largely mediated by tight binding of compstatin to plasma C3 (Qu et al., 2013). Whereas compstatin analogs with binding affinity in the sub-nanomolar range (i.e, Cp40) already show good pharmacokinetic profiles (Qu et al., 2013), making these agents suitable for sustained therapeutic intervention, elegant approaches have led to a further increase in plasma residence. These strategies include PEGylation approaches that have led to an impressive increase in Cp40's half-life (Risitano et al., 2014), or conjugation of Cp20 to plasma carrier proteins by means of small albumin-binding tags (Huang et al., 2014). Notably, one of the albumin-binding 
compstatin derivatives (ABM2-Cp20) displays a 20-fold higher binding affinity for $C 3\left(\mathrm{~K}_{\mathrm{D}}=150 \mathrm{pM}\right)$ than does the parental peptide, making it the most potent compstatin analog to date (Huang et al., 2014).

Their beneficial pharmacokinetic properties and suitability for subcutaneous administration make $\mathrm{Cp} 40$ and other next-generation analogs promising candidate drugs for C3 intervention in both acute and chronic indications driven by C3 dysregulation and derailed AP amplification (e.g., hemodialysis, PNH, C3G). AMY-101 (Amyndas), a C3-targeted therapeutic based on Cp40, shows a significant (6,000-fold) improvement in binding affinity to human C3 when compared to the original compstatin, as well as an enhanced inhibitory potency and extended in vivo half-life in NHP when compared to the much shorter half-lives of earlier compstatin analogs.

Overall, compstatin's structure-guided optimization has led to an impressive lineup of C3 therapeutics that display favorable pharmacokinetic profiles and sustained biological efficacy in a wide spectrum of indications. The therapeutic potential and medical plausibility of targeting native C3 with inhibitors of the compstatin family has recently been endorsed by international regulatory authorities. First-generation compstatin analogs (Potentia/Apellis) have received orphan status for PNH from the US Food and Drug Administration (FDA). Furthermore, a C3-targeted therapeutic based on next-generation compstatin analogs (i.e., AMY-101, Amyndas) has received orphan designation from both the European Medicines Agency (EMA) and the FDA for the treatment of PNH and C3G, two rare diseases etiologically linked to complement AP dysregulation [reviewed in (Ricklin and Lambris, 2015; Mastellos et al., 2015).

\section{2. "Pinning" the endogenous $\mathrm{C} 3$ regulators down to the surface}

Exploiting the body's own homeostatic circuitry of fluid-phase and surface-bound C3 regulators in order to modulate complement activation on diseased surfaces has illuminated new therapeutic directions in the complement field. Several groups have developed C3 therapeutics adopting the "opsonin-targeted" approach that essentially localizes potent complement inhibitors or regulators to sites of high opsonic turnover via specific molecular tethers (Holers et al., 2013;Ricklin and Lambris, 2016a). Given that C3 dysregulation is emerging as a key driver of pathophysiology in a number of inflammatory conditions, the surface-tethering of potent $\mathrm{C} 3$ convertase regulators, such as factor $\mathrm{H}(\mathrm{FH})$, and the engineering of fusion or miniaturized proteins encompassing inhibitory and surface-recognition modules of C3 regulators have led to attractive therapeutic leads in several AP-mediated disease models. 
FH-CR2 fusion proteins: The opsonin-targeted approach has provided important proof-of-concept for the therapeutic potential of targeting complement modulation to diseased (opsonized) surfaces. An illustrative and pioneering example has been the design of fusion proteins (e.g., TT30, Taligen; later developed by Alexion) encompassing the regulatory modules of the AP regulator FH (CCP domains 1-5), combined with the iC3b/C3dg-binding domains of complement receptor 2 (CR2; CCP1-4) (Fridkis-Hareli et al., 2011). Such chimeric regulators localize complement modulation to the C3-opsonized surface via their CR2 tether. In this regard, TT30 has shown therapeutic efficacy in AP-driven disease models (Banda et al., 2009; Holers et al., 2013) and has been clinically developed for the treatment of PNH. Whereas a Phase I trial of TT30 has proved successful in untreated PNH patients, further clinical development of this inhibitor has not yet been reported (Risitano et al., 2015). Interestingly, this therapeutic concept of opsonin targeting has been extended to other regulators such as CR1 (i.e., TT32) and has also inspired a diversified spectrum of chimeric inhibitors/regulators (e.g., FH-MAP1, FH-CRIg, and CD59-CRIg [reviewed in (Ricklin and Lambris, 2016b)]).

Mini-FH variants: Key structural insights concerning C3 convertase regulation have been integrated into a series of miniaturized versions of human $\mathrm{FH}(\mathrm{mini}-\mathrm{FH})$ with emerging clinical potential (Schmidt et al., 2013; Hebecker et al., 2013). Despite a significant reduction in size as compared to FH, these mini-FH molecules have retained the complement regulatory and surface-recognition capacity of the parental $\mathrm{FH}$ by directly combining CCP domains 1-4 and 19-20 respectively. Mini-FH exhibits an increased APtargeted inhibitory potency, binding C3-derived opsonic fragments (iC3b/C3dg) with a higher affinity than that of FH. This new class of surface-targeted inhibitors (e.g., AMY-201, Amyndas) has shown promising therapeutic results in an AP-driven disease model, protecting the erythrocytes of PNH patients from C3 opsonization and intravascular hemolysis (Schmidt et al., 2013). Notably, a recent study guided by molecular design has revealed distinct selectivity criteria that determine the relative inhibitory potency of surface-targeted C3 inhibitors on different C3-opsonized surfaces (Schmidt et al., 2016). In this context, mini-FH showed greater efficacy in abrogating complement activation on PNH erythrocytes, whereas other targeted regulator constructs showed distinct activities on different surfaces (Schmidt et al., 2016). These findings may better guide C3 therapeutic design by allowing us to tailor the application of surface-targeted inhibitors to appropriate pathologies.

Recruitment of FH to non-self surfaces: Once a biomaterial surface or xenogeneic tissue comes into contact with blood, it becomes exposed to complement-mediated opsonization and an ensuing 
thromboinflammatory response (Ekdahl et al., 2015). An evolutionarily conserved mechanism by which microbes evade complement attack is the decoration of their surface with proteins that can sequester FH from the host's circulation. This primordial immune evasion strategy has prompted the design of novel therapeutics that exploit targeting moieties such as peptides for recruiting fluid-phase C3 regulators to non-self or artificial surfaces (Ricklin and Lambris, 2016a). Recruitment of FH to cells or biomaterial surfaces coated with an FH-binding peptide (e.g., 5C6) has been shown to prevent AP-driven complement activation, attenuating blood-mediated thromboinflammatory reactions (Wu et al., 2011). Such C3 regulator-targeting moieties provide a new therapeutic handle for containing inflammatory complications in transplantation medicine or biomaterial-triggered pathologies (Ekdahl et al., 2015; Kourtzelis et al., 2015).

\section{Clinical advancement of compstatin-based C3 inhibitors: State-of-the-art}

As the list of clinical disorders etiologically linked to complement dysregulation grows longer, it is increasingly appreciated that complement modulation at the level of C3 may offer broad therapeutic benefit and, in some cases, even superior treatment options to patients failing to respond to standard anti-complement therapy. Clinical conditions that present an opportunity for C3-targeted intervention can be classified into acute (or transient) indications that may benefit from short-term or intermittent C3 interception and chronic indications that require long-term complement modulation. On their way to clinical translation, the most potent compstatin derivatives have shown promise as novel therapeutics in primate models of sepsis-related (Silasi-Mansat et al., 2010) and hemodialysis (HD)-induced inflammation (Reis et al., 2014) and in ex vivo models of xenotranslantation (Kourtzelis et al., 2014), and xenoantibody-mediated toxicity (Wang et al., 2016); they have also shown profound efficacy in models of AP-driven chronic debilitating diseases such as PNH and C3G (Risitano et al., 2014;Zhang et al., 2015). Notably, local application of the current lead analog, $\mathrm{Cp} 40$, has recently been shown to attenuate both inducible and naturally occurring periodontitis in NHP (Maekawa et al., 2014;Maekawa et al., 2016), opening up new prospects for C3-targeted clinical intervention in this prevalent dysbiotic oral inflammatory disease. While the application of compstatin analogs in NHP models has underscored the feasibility of therapeutic intervention at the level of C3 and provided important insights into disease pathogenesis, it will still be necessary to gain solid clinical experience by monitoring these C3-targeted therapeutics in future clinical trials. 


\subsection{Acute indications}

\section{Hemodialysis-induced thromboinflammation}

Exposure of biomaterial surfaces (e.g., implants, HD filters, or extracorporeal perfusion circuits) to whole blood constituents can rapidly trigger complement activation that will, in turn, fuel an vicious cycle of inflammation, leading to procoagulant responses that have detrimental consequences for organ function (Ekdahl et al., 2015). Recent advances in surface nanopatterning and biopolymer technology have attempted to bridge the gap of incompatibility in such systems, but nevertheless, clinical complications remain, largely as the result of recurring biomaterial-induced inflammatory episodes that exacerbate chronic underlying pathologies (Teramura et al., 2015). Hemodialysis has been associated with such thromboinflammatory responses, which likely increase the burden of disease (e.g., risk of cardiovascular disease). Patients suffering from end-stage renal disease (ESRD) are often forced to rely for many years on periodic sessions of hemodialysis (HD) because of their deterioration of kidney function and the shortage of donor replacement organs (Santoro et al., 2013). Interestingly, studies have shown that even modern "biocompatible" HD filters can trigger appreciable levels of complement activation and also induce TF expression, thereby contributing to a thromboinflammatory milieu that can increase morbidity in ESRD patients (Kourtzelis et al., 2010). Notably, this thromboinflammatory reaction has been shown to be attenuated ex vivo by the earlier compstatin analog $4(1 \mathrm{MeW})$ (Kourtzelis et al., 2010). Recently, these findings were recapitulated in a refined, clinically relevant NHP model of HD-induced inflammation (Reis et al., 2014). Cynomolgus monkeys were subjected to HD using a circuit of pediatric hemodialysis filters, and significant complement activation was observed even after a single HD session. Of particular note, a single intravenous bolus injection of compstatin Cp40 prior to HD completely abrogated HD-induced complement activation and elevated levels of the anti-inflammatory cytokine IL-10 (Reis et al., 2014). The time-restricted dosing scheme of Cp40 in hemodialysis allows for rapid recovery of complement activity between sessions, thereby pointing to a safe and affordable treatment option that may alleviate inflammatory complications in ESRD patients. Furthermore, the availability of surface-targeting moieties and coating strategies that can direct soluble C3 regulators to surfaces (Wu et al., 2011) offers alternative routes for modulating complement activation and containing inflammatory responses evoked by HD filters and other medically applied biomaterial surfaces (e.g., biomedical implants). 


\section{C3 intervention in transplantation}

Transplantation medicine encompasses a vibrant area of investigation that has recently opened up new opportunities for C3-targeted intervention. Complement activation is increasingly recognized as a key pathogenic driver in acute antibody-mediated rejection (AMR) following allogeneic organ transplantation (Sacks and Zhou, 2012;Stegall et al., 2012), and its role in chronic organ rejection is also under debate. An initial trigger for this tissue-destructive process is the sensitization of transplant recipients by donor-specific alloantibodies (i.e., HLA and ABO transplantation barriers) (Biglarnia et al., 2012). Therefore, complement inhibitors targeting either early or late components of the cascade have gained momentum as potential therapeutic leads for graft accommodation and long-term survival (Sacks and Zhou, 2012).

Therapeutic modulation at the level of $\mathrm{C} 5$ has shown promise in alleviating complications of early AMR and prolonging kidney graft survival. However, a fair proportion of graft recipients fail to respond to anti-C5 treatment, implying that a broader and more comprehensive intervention might be warranted (Bentall et al., 2014). In this regard, C3 interception is considered a plausible approach for improving graft accommodation and function in transplant recipients, given its multifaceted impact on both cell-mediated and humoral inflammatory pathways. In fact, proof of concept for a role of C3 in promoting graft accommodation in renal transplantation has been provided in an NHP renal transplant model employing CVF as a complement-depleting agent (Chen et al., 2011). In addition, earlier compstatin analogs have shown efficacy in abrogating complement activation and cell-mediated inflammation in ex vivo xenotransplantation (xeno-Tx) models (Fiane et al., 1999;Goto et al., 2008). Indeed, the sustained inhibitory potency and clinical potential of next-generation compstatin derivatives make them attractive treatment options for attenuating inflammatory complications in organ transplantation (Mastellos et al., 2015). Along these lines, the Cp40-based inhibitor AMY-101 (Amyndas Pharmaceuticals) is currently under clinical development as a therapeutic for improving graft accommodation in ABO-incompatible kidney transplantation (Mastellos et al., 2015).

In addition to the transplantation of ABO- or HLA-incompatible organs, xeno-Tx has also been proposed as a countermeasure to the rapidly escalating shortage of donor organs (Kourtzelis et al., 2015). However, hyperacute rejection resulting from the instant thromboinflammatory responses to the xenogeneic tissue is a major hurdle in applying xeno-Tx in the clinic. Recent studies have highlighted the therapeutic potential of C3-targeted interception in this emerging clinical field. Indeed, compstatin analogs have shown promising results, abrogating complement activation and thromboinflammatory 
cell activation in a primate model of islet xeno-Tx (Goto et al., 2008), a whole-blood model of xenogeneic interactions between human blood and porcine endothelium (Kourtzelis et al., 2014), and in a porcine-to- human model of xenoantibody-induced complement-dependent cytotoxicity (Wang et al., 2016) .

\subsection{Chronic indications}

Paroxysmal nocturnal hemoglobinuria (PNH)

$\mathrm{PNH}$ is a rare hematological disorder genetically driven by a deficiency of the GPI-anchored proteins CD55 and CD59 on erythrocytes that leads to chronic AP dysregulation and MAC-mediated intravascular hemolysis (Takeda et al., 1993;Risitano, 2012). PNH is consistently characterized by three clinical hallmarks: hemolytic anemia, bone marrow failure, and thrombophilia (Parker et al., 2005). Given its prominent linkage to complement dysregulation, PNH has long been considered an ideal paradigm for complement-based therapeutic intervention (Risitano, 2012). The advent of anti-C5 therapy (i.e., eculizumab) has significantly improved the treatment landscape of PNH (Rother et al., 2007). By abrogating intravascular hemolysis, this complement-targeted drug has brought clinical benefit to $70 \%$ of PNH patients; however, a significant fraction of patients receiving anti-C5 are either suboptimal responders or are refractory to therapy because of complement-related genetic variance and still require blood transfusions to treat their residual anemia (Risitano et al., 2010;Nishimura et al., 2014). Interestingly, anti-C5 treatment has unmasked a novel pathogenic mechanism that explains the limited hematologic benefit afforded to certain PNH patients (Risitano et al., 2009): Persistent C3-mediated opsonization of surviving PNH RBCs coupled to poorly controlled AP amplification, resulting from the genetic absence of GPI-linked CD55, may lead to C3-mediated extravascular hemolysis and chronic residual anemia (Risitano et al., 2009). Recent in vitro studies have corroborated this clinical observation by showing that C3dg-opsonized RBCs from eculizumab-treated PNH patients are recognized and efficiently phagocytosed by macrophages (Lin et al., 2015).

C3 interception has therefore emerged as a promising strategy for controlling the entire spectrum of detrimental consequences evoked by complement dysregulation in PNH (Mastellos et al., 2014). In this respect, peptidic C3 inhibitors with pharmacokinetic profiles amenable to systemic administration are currently being explored. The compstatin analog $\mathrm{Cp} 40$ has shown efficacy in abrogating MAC-mediated hemolysis of PNH erythrocytes and also in preventing AP-amplified C3 
opsonization (Risitano et al., 2014). From a different standpoint, C3 regulator-based approaches have also illustrated the clinical potential of targeting AP amplification closer to the C3-opsonized surface of PNH cells (Risitano et al., 2012;Schmidt et al., 2013). Collectively, these studies indicate that C3 interception may afford greater therapeutic benefit to PNH patients than that achieved by anti-C5 therapy, by also mitigating C3-mediated extravascular hemolysis (Mastellos et al., 2014). The favorable pharmacokinetic and safety profiles and the sustained efficacy of Cp40/AMY-101 in NHP models attest to the feasibility of chronic administration of $\mathrm{C} 3$ inhibitors in PNH patients. Of note, the projected annual costs of the standard anti-C5 therapy impose a considerable economic burden on the healthcare system, likely limiting broader access of patients to this effective treatment option, particularly in developing countries. Small-sized therapeutics such as compstatin analogs may present one potential avenue towards more affordable PNH therapies. Moreover, a different mode of complement inhibition at the level of C3, which can effectively contain both intravascular and extravascular hemolysis, may potentially result in broader clinical efficacy in at least the subgroup of PNH patients that show insufficient response to established treatment. Future clinical trials to validate this new therapeutic option in PNH patients are highly anticipated.

\section{Periodontal disease}

Periodontitis is a chronic oral inflammatory disease that culminates in the destruction of the toothsupporting connective and bone tissue (Hajishengallis, 2014a). Inflammation induced and perpetuated by dysbiotic bacterial communities in subgingival tooth sites is the main pathogenic driver of the disease. Chronic periodontitis affects $>47 \%$ of U.S. adults (Eke et al., 2012), and its most severe form is associated with increased risk for certain systemic disorders (e.g., atherosclerosis, diabetes, rheumatoid arthritis, and adverse pregnancy outcomes) (Hajishengallis, 2015). The serious public health impact of periodontitis and its economic burden (Beikler and Flemmig, 2011;Chapple, 2014) require effective and affordable therapeutic options capable of improving on existing therapies (mechanical removal of the tooth-associated biofilm and antimicrobial treatment), which are often insufficient to control periodontitis (Colombo et al., 2012;Armitage, 2002;Rams et al., 2014).

Recent studies have provided compelling evidence that local complement activation, in synergy with TLRs, promotes dysbiosis while exacerbating the resulting inflammation and bone loss in periodontitis [reviewed in (Hajishengallis et al., 2016)]. Indeed, genetic and pharmacological studies have shown that both C3 and C5aR1 are targets for therapeutic intervention in models of periodontal disease (Abe et al., 
2012;Maekawa et al., 2014). In view of the broader therapeutic benefit afforded by upstream C3 targeting in many disease models, the peptidic C3 inhibitor Cp40 has been evaluated as a therapeutic option in an NHP model of ligature-induced periodontitis. Intragingival treatment of affected monkeys with Cp40 resulted in attenuation of periodontal inflammation (as evidenced by decreased clinical indices that measure periodontal inflammation and tissue destruction) and protection from bone loss over a 6-week period (Maekawa et al., 2014). These protective effects were correlated with lower levels of inflammatory and bone resorptive mediators (e.g., TNF, IL-1 $\beta$, IL-17) in gingival crevicular fluid and decreased osteoclastogenesis (e.g., RANKL expression) in bone biopsies. Extending these observations to a more clinically relevant context, Maekawa and colleagues recently showed that local administration of Cp40 can effectively reverse the signs of periodontitis (gingival inflammation and osteoclastogenesis) in an NHP model of chronic, naturally-occurring periodontitis (Maekawa et al., 2016). Notably, even a single weekly injection of $\mathrm{Cp} 40$ for 6 weeks caused a marked reduction in a panel of clinical indices and laboratory tests reflecting periodontal inflammation and bone resorption. This protective effect could be maintained for a prolonged period after discontinuation of therapy ( 6 weeks), likely indicating long-term effects of C3 inhibition. Taken together, these findings underscore the clinical potential of Cp40-based therapeutics, such as AMY-101, in dysbiotic oral inflammation and pave the way for C3-based immunomodulatory therapies for the treatment of human periodontitis.

Important questions that remain to be addressed include the most suitable and effective formulation and dosing scheme and the optimal therapeutic window to prevent or treat periodontal inflammation and bone loss.

\section{C3 glomerulopathy}

C3 glomerulopathy (C3G) is a recently adopted term that collectively defines a group of ultra-rare renal disorders characterized by pronounced $\mathrm{C} 3$ deposition in the renal tissue (i.e., glomeruli), in the absence (or scarce presence) of deposited immunoglobulins (Pickering et al., 2013). C3G is subdivided into two pathological entities: dense deposit disease (DDD), which encompasses renal pathologies characterized by highly electron-dense deposits, and C3 glomerulonephritis (C3GN) which describes glomerular lesions with pronounced C3 deposition, but lacking the characteristic highly electron-dense transformation (Pickering et al., 2013; Sethi and Fervenza, 2014). Half of all C3G patients progress to end-stage renal failure within 10 years of diagnosis, and allograft rejection occurs following kidney transplantation secondary to disease relapse in more than $50 \%$ of transplant recipients. Pathology in C3G patients is largely driven by genetic or acquired AP dysregulation (Pickering et al., 2013; Servais et 
al., 2012). Genetic factors predisposing to C3G include mutations in a wide spectrum of genes encoding AP components and regulators (e.g., C3, FH, FI, FB) (Servais et al., 2012). The complexity of the genetic landscape underlying complement dysregulation in C3G is underscored by recently reported chromosomal aberrations in the complement FH-related (CFHR) gene cluster yielding CFHR fusion proteins that might deregulate C3 convertase activity (Chen et al., 2014). Interestingly, a founder mutation in the CFHR5 gene that segregates with families of Cypriot origin has also been implicated with a form of C3G (CFHR5 nephropathy) (Gale et al., 2010). C3G is also associated with the presence of autoantibodies that function as acquired drivers of AP dysregulation. These autoantibodies, termed C3 nephritic factors (C3Nefs), bind to C3 convertases to prevent their FH-mediated decay, thereby prolonging their half-lives and leading to chronic AP dysregulation (Xiao et al., 2014).

Currently, there is no treatment available for C3G. Management is mostly palliative and includes drugs for blood pressure control and immunosuppressive therapy (Xiao X, et al. 2014). Since APmediated complement dysregulation is the main pathological driver of C3G, C3-targeted intervention has emerged as a promising strategy for mitigating pathological sequelae in the renal tissue and improving therapeutic outcomes in C3G patients. Proof-of-concept for the therapeutic potential and safety of C3 intervention in C3G was recently provided by a study documenting the efficacy of a soluble form of CR1 in a pediatric DDD patient (Zhang et al., 2013). The favorable pharmacokinetic profiles and sustained potency of C3 inhibitors of the compstatin family make them attractive and affordable drug leads for the treatment of C3G. Indeed, Cp40 has shown consistent efficacy in reverting complement dysregulation in in vitro models of C3G (Zhang et al., 2015). This C3-targeted inhibitor can suppress complement-mediated hemolysis in the sera of C3G patients and reverses complement dysregulation caused by patient-derived autoantibodies. Moreover, treatment with $\mathrm{Cp} 40$ prevents complement dysregulation associated with C3G-predisposing genetic mutations, suggesting a wider therapeutic impact in both acquired and genetically driven C3G. These findings not only pave the way for a targeted, disease-specific therapy for C3G but also open up new prospects for a broad spectrum of C3 therapeutics that can modulate AP activity, both in the fluid phase and closer to the opsonized surface. Endorsing the clinical potential of C3-targeted inhibitors, both the EMA and FDA have accorded the C3 therapeutic AMY-101 an orphan designation for the treatment of C3G (AMYNDAS Pharmaceuticals, 2016). Notably, AMY-101 is the first complement-targeted drug to receive orphan designation for this indication. 


\section{Translational considerations and future outlook}

Translating preclinical findings to the patient's bedside is a multifaceted process that goes through several clinical and regulatory checkpoints. Furthermore, the projected therapeutic benefit of any complement-targeted therapy must always be weighed against the potential risks, and effective mitigation measures should be integrated into the designed protocol. Along an intensive course of preclinical development, peptidic C3 inhibitors of the compstatin family have overcome certain concerns often raised with systemic C3 interception and peptide drug development. Such concerns have mostly revolved around issues of target saturation, plasma stability, feasibility of prolonged complement modulation, pharmacokinetics, and pathogen immunosurveillance during intervention (Ricklin and Lambris, 2015). As exemplified by next-generation compstatin analogs, saturable binding to plasma C3 can be achieved in conjunction with slower plasma elimination rates that are largely driven by a subnanomolar affinity-binding to C3 (Qu et al., 2013). Moreover, a highly favourable pharmacokinetic behavior and sustained inhibitory potency have been observed after subcutaneous (SQ) administration of these C3 inhibitors (Risitano et al., 2014). This route of administration may offer increased patient compliance in chronic protocols of C3 intervention that require frequent dosing. Future studies will still have to explore alternative routes of administration or tailored formulations that may afford greater therapeutic benefit in a disease-specific context.

With regard to chronic indications, long-term C3 inhibition justifiably stirs discussions about the maintenance of pathogen immune surveillance. While most of these concerns are sparked by the increased susceptibility of C3-deficient individuals to infections by encapsulated bacteria, interpretation can still be rather contextual, as it is not yet clear how directly these clinical observations might translate to a therapeutic setting using C3 inhibitors. Of note, clinical experience from primary C3 deficiencies indicates that patients are more susceptible to opportunistic infections in the earlier stages of life, whereas this susceptibility later subsides during adulthood, implying the recruitment of compensatory mechanisms for long-term immunity (Reis et al., 2006). However, given the pivotal role of various complement proteins (e.g., C3 and C5) in the phagocytic clearance of microbial intruders, a treatment scheme including vaccination should always be considered alongside prolonged C3 or C5 inhibitory protocols. Whereas in the clinical practice chronic anti-C5 therapy mandates meningococcal vaccination, additional prophylaxis against highly virulent encapsulated bacteria, such as pneumococci or Haemophilus influenza, may need to be considered during prolonged C3 intervention. Acute or transient C3 interception, in indications such as hemodialysis or transplantation, is not expected to raise any 
significant safety concerns, while local application of C3-targeted inhibitors (as in the case of periodontal diseases) may even prove beneficial for the local containment of bacterial growth (Hajishengallis et al, 2016), since periodontal dysbiotic microbial communities thrive on destructive inflammation, which generates nutrients such as collagen peptides and heme-containing compounds via tissue breakdown (Hajishengallis, 2014b). Of particular note, anti-C3 treatment with small-sized inhibitors (such as AMY101/Cp40) can be readily interrupted in a clinical protocol, allowing for swift recovery of C3 activity during an infection. Whereas concerns relating to a purportedly increased risk of autoimmune reactions or immune complex-mediated inflammatory responses during long-term C3 intervention have yet to be validated by concrete evidence (reviewed in (Mastellos et al., 2015), these issues will have to be carefully monitored during future clinical trials. As with all clinical protocols that target immunomodulatory systems, safety should always be reconciled with the projected therapeutic benefit of the treatment in question. In this respect, the potential safety concerns raised by long-term C3 intervention and their associated risk-mitigating measures have been extensively reviewed (Mastellos et al, 2015).

Undeniably, the complement field is witnessing the assembly of an unprecedented lineup of therapeutics targeting various steps of the cascade and catering for different clinical indications. In this respect, C3-targeted therapeutics have gained considerable momentum through a surge of creative approaches exploiting both fluid-phase and surface-tethered inhibitors for therapeutic C3 modulation. In light of the emerging evidence that population-wide genetic variance intricately influences patient responses to anti-complement therapy, future clinical trials will ultimately decide on the translational value of these inhibitors as stand-alone or adjunct immunotherapeutics for complement-mediated diseases. 


\section{Acknowledgments}

We thank Dr. Deborah McClellan for editorial assistance. The authors are supported by grants from the U.S. National Institutes of Health (AI003040, AI068730), the National Science Foundation (No. 1423304), and the European Community's Seventh Framework Programme under grant agreement number 602699 (DIREKT) (J.D.L.) and DE015254, DE021685, and DE024716 (G.H.). 


\section{References}

1. Abe,T., Hosur,K.B., Hajishengallis,E., Reis,E.S., Ricklin,D., Lambris,J.D., Hajishengallis,G., $2012 . \quad$ Local complement-targeted intervention in periodontitis: proof-of-concept using a C5a receptor (CD88) antagonist. J. Immunol. 189, 5442-5448.

2. Alcon Research. A Multicenter, Proof-of-Concept Study of Intravitreal AL-78898A in Patients with Geograhic Atrophy (GA) associated with Age-Related Macular Degeneration (AMD). ClinicalTrials.gov, Identifier:NCT01603043 . 2013.

3. AMYNDAS Pharmaceuticals. Amyndas' lead candidate AMY-101 receives orphan drug status from the FDA and the EMA for the treatment of C3 glomerulopathy. http://www.fiercepharma.com/pharma/amyndas\%E2\%80\%99-lead-candidate-amy-101-receives-orphandrug-status-from-fda-and-ema-for-treatment . 4-14-2016.

4. Armitage,G.C., 2002. Classifying periodontal diseases--a long-standing dilemma. Periodontol. 2000. 30, 9-23.

5. Banda,N.K., Levitt,B., Glogowska,M.J., Thurman,J.M., Takahashi,K., Stahl,G.L., Tomlinson,S., Arend,W.P., Holers,V.M., 2009. Targeted inhibition of the complement alternative pathway with complement receptor 2 and factor $\mathrm{H}$ attenuates collagen antibody-induced arthritis in mice. J. Immunol. 183, 5928-5937.

6. Beikler,T., Flemmig,T.F., 2011. Oral biofilm-associated diseases: trends and implications for quality of life, systemic health and expenditures. Periodontol. 2000. 55, 87-103.

7. Bentall,A., Tyan,D.B., Sequeira,F., Everly,M.J., Gandhi,M.J., Cornell,L.D., Li,H., Henderson,N.A., Raghavaiah,S., Winters,J.L., Dean,P.G., Stegall,M.D., 2014. Antibody-mediated rejection despite inhibition of terminal complement. Transpl. Int., 27, 1235-43.

8. Berentsen,S., 2015. Role of Complement in Autoimmune Hemolytic Anemia. Transfus. Med. Hemother. 42, 303-310.

9. Biglarnia,A.R., Nilsson,B., Nilsson,E.K., Tufveson,G., Nilsson,T., Larsson,E., Wadstrom,J., 2012. Desensitization with antigen-specific immunoadsorption interferes with complement in ABO-incompatible kidney transplantation. Transplantation 93, 87-92.

10. Chapple,I.L., 2014. Time to take periodontitis seriously. BMJ 348, g2645.

11. Chen,Q., Wiesener,M., Eberhardt,H.U., Hartmann,A., Uzonyi,B., Kirschfink,M., Amann,K., Buettner,M., Goodship,T., Hugo,C., Skerka,C., Zipfel,P.F., 2014. Complement factor H-related hybrid protein deregulates complement in dense deposit disease. J. Clin. Invest 124, 145-155.

12. Chen,S.S., Zhong,S., Xiang,Y., Li,J.H., Guo,H., Wang,W.Y., Xiong,Y.L., Li,X.C., Chen,S.S., Chen,X.P., Chen,G., 2011. Complement inhibition enables renal allograft accommodation and long-term engraftment in presensitized nonhuman primates. Am. J. Transplant. 11, 2057-2066. 
13. Chi,Z.L., Yoshida,T., Lambris,J.D., Iwata,T., 2010. Suppression of drusen formation by compstatin, a peptide inhibitor of complement C3 activation, on cynomolgus monkey with early-onset macular degeneration. Adv. Exp. Med. Biol. 703, 127-135.

14. Colombo,A.P., Bennet,S., Cotton,S.L., Goodson,J.M., Kent,R., Haffajee,A.D., Socransky,S.S., Hasturk,H., Van Dyke,T.E., Dewhirst,F.E., Paster,B.J., 2012. Impact of periodontal therapy on the subgingival microbiota of severe periodontitis: comparison between good responders and individuals with refractory periodontitis using the human oral microbe identification microarray. J. Periodontol. 83, 1279-1287.

15. Coulthard LG1, Woodruff T.M., 2015. Is the complement activation product C3a a proinflammatory molecule? Re-evaluating the evidence and the myth. J Immunol. 194, 3542-8.

16. Ekdahl,K.N., Lambris,J.D., Elwing,H., Ricklin,D., Nilsson,P.H., Teramura,Y., Nicholls,I.A., Nilsson,B., 2011. Innate immunity activation on biomaterial surfaces: a mechanistic model and coping strategies. Adv. Drug Deliv. Rev. 63, 1042-1050.

17. Ekdahl,K.N., Teramura,Y., Asif,S., Jonsson,N., Magnusson,P.U., Nilsson,B., 2015. Thromboinflammation in Therapeutic Medicine. Adv. Exp. Med. Biol. 865, 3-17.

18. Eke,P.I., Dye,B.A., Wei,L., Thornton-Evans,G.O., Genco,R.J., 2012. Prevalence of periodontitis in adults in the United States: 2009 and 2010. J. Dent. Res. 91, 914-920.

19. Fiane AE, Mollnes TE, Videm V, Hovig T, Høgåsen K, Mellbye OJ, Spruce L, Moore WT, Sahu A, Lambris JD., 1999. Compstatin, a peptide inhibitor of C3, prolongs survival of ex vivo perfused pig xenografts. Xenotransplantation 6: 52-65.

20. Fridkis-Hareli,M., Storek,M., Mazsaroff,I., Risitano,A.M., Lundberg,A.S., Horvath,C.J., Holers,V.M., 2011. Design and development of TT30, a novel C3d-targeted C3/C5 convertase inhibitor for treatment of human complement alternative pathway-mediated diseases. Blood 118, 4705-4713.

21. Gale,D.P., de Jorge,E.G., Cook,H.T., Martinez-Barricarte,R., Hadjisavvas,A., McLean,A.G., Pusey,C.D., Pierides,A., Kyriacou,K., Athanasiou,Y., Voskarides,K., Deltas,C., Palmer,A., Fremeaux-Bacchi,V., de Cordoba,S.R., Maxwell,P.H., Pickering,M.C., 2010. Identification of a mutation in complement factor $\mathrm{H}$ related protein 5 in patients of Cypriot origin with glomerulonephritis. Lancet 376, 794-801.

22. Goto,M., Tjernberg,J., Dufrane,D., Elgue,G., Brandhorst,D., Ekdahl,K.N., Brandhorst,H., Wennberg,L., Kurokawa,Y., Satomi,S., Lambris,J.D., Gianello,P., Korsgren,O., Nilsson,B., 2008. Dissecting the instant blood-mediated inflammatory reaction in islet xenotransplantation. Xenotransplantation. 15, $225-234$.

23. Gros,P., Milder,F.J., Janssen,B.J., 2008. Complement driven by conformational changes. Nat. Rev. Immunol. 8, 48-58.

24. Hajishengallis,G., 2014a. Immunomicrobial pathogenesis of periodontitis: keystones, pathobionts, and host response. Trends Immunol. 35, 3-11.

25. Hajishengallis,G., 2014b. The inflammophilic character of the periodontitis-associated microbiota. Mol. Oral Microbiol. 29, 248-257. 
26. Hajishengallis,G., 2015. Periodontitis: from microbial immune subversion to systemic inflammation. Nat. Rev. Immunol. 15, 30-44.

27. Hajishengallis,G., Hajishengallis,E., Kajikawa,T., Wang,B., Yancopoulou,D., Ricklin,D., Lambris,J.D., 2016. Complement inhibition in pre-clinical models of periodontitis and prospects for clinical application. Semin. Immunol. [In Press]

28. Harboe M, Ulvund G, Vien L, Fung M, Mollnes TE., 2004. The quantitative role of alternative pathway amplification in classical pathway induced terminal complement activation. Clin Exp Immunol. 138, 43946.

29. Harboe M, Garred P, Karlstrøm E, Lindstad JK, Stahl GL, Mollnes TE., 2009. The down-stream effects of mannan-induced lectin complement pathway activation depend quantitatively on alternative pathway amplification. Mol Immunol. 47, 373-80.

30. Harris,C.L., Heurich,M., Rodriguez de,C.S., Morgan,B.P., 2012. The complotype: dictating risk for inflammation and infection. Trends Immunol. 33, 513-521.

31. Hebecker,M., Alba-Dominguez,M., Roumenina,L.T., Reuter,S., Hyvarinen,S., Dragon-Durey,M.A., Jokiranta,T.S., Sanchez-Corral,P., Jozsi,M., 2013. An engineered construct combining complement regulatory and surface-recognition domains represents a minimal-size functional factor $\mathrm{H}$. J. Immunol. 191, 912-921.

32. Holers,V.M., Rohrer,B., Tomlinson,S., 2013. CR2-mediated targeting of complement inhibitors: bench-tobedside using a novel strategy for site-specific complement modulation. Adv. Exp. Med. Biol. 735, 137154.

33. Huang,Y., Reis,E.S., Knerr,P.J., van der Donk,W.A., Ricklin,D., Lambris,J.D., 2014. Conjugation to albuminbinding molecule tags as a strategy to improve both efficacy and pharmacokinetic properties of the complement inhibitor compstatin. ChemMedChem. 9, 2223-2226.

34. Huber-Lang,M., Sarma,J.V., Zetoune,F.S., Rittirsch,D., Neff,T.A., McGuire,S.R., Lambris,J.D., Warner,R.L., Flierl,M.A., Hoesel,L.M., Gebhard,F., Younger,J.G., Drouin,S.M., Wetsel,R.A., Ward,P.A., 2006. Generation of C5a in the absence of C3: a new complement activation pathway. Nat. Med. 12, 682-687.

35. Janssen,B.J., Halff,E.F., Lambris,J.D., Gros,P., 2007. Structure of compstatin in complex with complement component C3c reveals a new mechanism of complement inhibition. J. Biol. Chem. 282, 29241-29247.

36. Katschke,K.J., Jr., Wu,P., Ganesan,R., Kelley,R.F., Mathieu,M.A., Hass,P.E., Murray,J., Kirchhofer,D., Wiesmann,C., van Lookeren,C.M., 2012. Inhibiting alternative pathway complement activation by targeting the factor D exosite. J. Biol. Chem. 287, 12886-12892.

37. Kolev,M., Friec,G.L., Kemper,C., 2014. Complement - tapping into new sites and effector systems. Nat. Rev. Immunol. 14, 811-20. 
38. Kourtzelis,I., Ferreira,A., Mitroulis,I., Ricklin,D., Bornstein,S.R., Waskow,C., Lambris,J.D., Chavakis,T., 2015. Complement Inhibition in a Xenogeneic Model of Interactions Between Human Whole Blood and Porcine Endothelium. Horm. Metab Res. 47, 36-42.

39. Kourtzelis,I., Magnusson,P.U., Kotlabova,K., Lambris,J.D., Chavakis,T., 2015. Regulation of Instant Blood Mediated Inflammatory Reaction (IBMIR) in Pancreatic Islet Xeno-Transplantation: Points for Therapeutic Interventions. Adv. Exp. Med. Biol. 865, 171-188.

40. Kourtzelis,I., Markiewski,M.M., Doumas,M., Rafail,S., Kambas,K., Mitroulis,I., Panagoutsos,S., Passadakis,P., Vargemezis,V., Magotti,P., Qu,H., Mollnes,T.E., Ritis,K., Lambris,J.D., 2010. Complement anaphylatoxin C5a contributes to hemodialysis-associated thrombosis. Blood 116, 631-639.

41. Lin,Z., Schmidt,C.Q., Koutsogiannaki,S., Ricci,P., Risitano,A.M., Lambris,J.D., Ricklin,D., 2015. Complement C3dg-mediated erythrophagocytosis: implications for paroxysmal nocturnal hemoglobinuria. Blood. 126, 891-4.

42. Maekawa,T., Abe,T., Hajishengallis,E., Hosur,K.B., Deangelis,R.A., Ricklin,D., Lambris,J.D., Hajishengallis,G., 2014. Genetic and intervention studies implicating complement c3 as a major target for the treatment of periodontitis. J. Immunol. 192, 6020-6027.

43. Maekawa,T., Briones,R.A., Resuello,R.R., Tuplano,J.V., Hajishengallis,E., Kajikawa,T., Koutsogiannaki,S., Garcia,C.A., Ricklin,D., Lambris,J.D., Hajishengallis,G., 2016. Inhibition of pre-existing natural periodontitis in non-human primates by a locally administered peptide inhibitor of complement C3. J. Clin. Periodontol. 43, 238-249.

44. Mastellos,D.C., Ricklin,D., Yancopoulou,D., Risitano,A., Lambris,J.D., 2014. Complement in paroxysmal nocturnal hemoglobinuria: exploiting our current knowledge to improve the treatment landscape. Expert. Rev. Hematol. 7, 583-598.

45. Mastellos,D.C., Yancopoulou,D., Kokkinos,P., Huber-Lang,M., Hajishengallis,G., Biglarnia,A.R., Lupu,F., Nilsson,B., Risitano,A.M., Ricklin,D., Lambris,J.D., 2015. Compstatin: a C3-targeted complement inhibitor reaching its prime for bedside intervention. Eur. J. Clin. Invest 45, 423-440.

46. Morgan,B.P., Harris,C.L., 2015. Complement, a target for therapy in inflammatory and degenerative diseases. Nat. Rev. Drug Discov. 14, 857-877.

47. Nishimura,J., Yamamoto,M., Hayashi,S., Ohyashiki,K., Ando,K., Brodsky,A.L., Noji,H., Kitamura,K., Eto,T., Takahashi,T., Masuko,M., Matsumoto,T., Wano,Y., Shichishima,T., Shibayama,H., Hase,M., Li,L., Johnson,K., Lazarowski,A., Tamburini,P., Inazawa,J., Kinoshita,T., Kanakura,Y., 2014. Genetic variants in C5 and poor response to eculizumab. N. Engl. J. Med. 370, 632-639.

48. Parker,C., Omine,M., Richards,S., Nishimura,J., Bessler,M., Ware,R., Hillmen,P., Luzzatto,L., Young,N., Kinoshita,T., Rosse,W., Socie,G., 2005. Diagnosis and management of paroxysmal nocturnal hemoglobinuria. Blood 106, 3699-3709. 
49. Pickering,M.C., D'Agati,V.D., Nester,C.M., Smith,R.J., Haas,M., Appel,G.B., Alpers,C.E., Bajema,I.M., Bedrosian,C., Braun,M., Doyle,M., Fakhouri,F., Fervenza,F.C., Fogo,A.B., Fremeaux-Bacchi,V., Gale,D.P., Goicoechea de,J.E., Griffin,G., Harris,C.L., Holers,V.M., Johnson,S., Lavin,P.J., Medjeral-Thomas,N., Paul,M.B., Nast,C.C., Noel,L.H., Peters,D.K., Rodriguez de,C.S., Servais,A., Sethi,S., Song,W.C., Tamburini,P., Thurman,J.M., Zavros,M., Cook,H.T., 2013. C3 glomerulopathy: consensus report. Kidney Int. 84, 10791089.

50. Qu,H., Ricklin,D., Bai,H., Chen,H., Reis,E.S., Maciejewski,M., Tzekou,A., Deangelis,R.A., Resuello,R.R., Lupu,F., Barlow,P.N., Lambris,J.D., 2013. New analogs of the clinical complement inhibitor compstatin with subnanomolar affinity and enhanced pharmacokinetic properties. Immunobiology 218, 496-505.

51. Qu,H., Ricklin,D., Lambris,J.D., 2009. Recent developments in low molecular weight complement inhibitors. Mol. Immunol. 47, 185-195.

52. Ra Pharmaceuticals. Ra Pharmaceuticals Announces Initiation of First-in-Human Clinical Trial of Novel Complement C5 Inhibitor. http://www.rapharma.com/global/downloads/Ra-Pharma-PR-120115-Phase-1ASH-FINAL.pdf . 4-14-2016.

53. Rams,T.E., Degener,J.E., van Winkelhoff,A.J., 2014. Antibiotic resistance in human chronic periodontitis microbiota. J. Periodontol. 85, 160-169.

54. Regillo C.D., Yaspan B.L, Li Z, Dressen A., van Lookeren Campagne M., Graham R., Beres T., Le K., Henry E., Ho C., Strauss E.C. On behalf of the MAHALO Study Investigators. Lampalizumab (Anti-factor D) in Patients with Geographic Atrophy: the MAHALO Phase II Results. 117th Annual AAO meeting. 117th Annual AAO meeting . 2013. New Orleans, LA.

55. Reis,E.S., Deangelis,R.A., Chen,H., Resuello,R.R., Ricklin,D., Lambris,J.D., 2014. Therapeutic C3 inhibitor Cp40 abrogates complement activation induced by modern hemodialysis filters. Immunobiology 220, 47682.

56. Reis,E.S., Mastellos,D.C., Yancopoulou,D., Risitano,A.M., Ricklin,D., Lambris,J.D., $2015 . \quad$ Applying complement therapeutics to rare diseases. Clin. Immunol. 161, 225-240.

57. Reis,S., Falcao,D.A., Isaac,L., 2006. Clinical aspects and molecular basis of primary deficiencies of complement component C3 and its regulatory proteins factor I and factor H. Scand. J. Immunol. 63, 155168.

58. Ricklin,D., Hajishengallis,G., Yang,K., Lambris,J.D., 2010. Complement: a key system for immune surveillance and homeostasis. Nat. Immunol. 11, 785-797.

59. Ricklin,D., Lambris,J.D., 2008. Compstatin: a complement inhibitor on its way to clinical application. Adv. Exp. Med. Biol. 632, 273-292.

60. Ricklin,D., Lambris,J.D., 2013a. Complement in immune and inflammatory disorders: pathophysiological mechanisms. J. Immunol. 190, 3831-3838. 
61. Ricklin,D., Lambris,J.D., 2013b. Complement in immune and inflammatory disorders: therapeutic interventions. J. Immunol. 190, 3839-3847.

62. Ricklin,D., Lambris,J.D., 2013c. Progress and trends in complement therapeutics. Adv. Exp. Med. Biol. 735, $1-22$.

63. Ricklin,D., Lambris,J.D., 2016a. Therapeutic control of complement activation at the level of the central component C3. Immunobiology. 221, 740-6.

64. Ricklin,D., Lambris,J.D., 2016b. New milestones ahead in complement-targeted therapy. Semin. Immunol. [In Press]

65. Risitano,A.M., 2012. Paroxysmal nocturnal hemoglobinuria and other complement-mediated hematological disorders. Immunobiology 217, 1080-1087.

66. Risitano,A.M., 2013. Paroxysmal nocturnal hemoglobinuria and the complement system: recent insights and novel anticomplement strategies. Adv. Exp. Med. Biol. 735, 155-172.

67. Risitano,A.M., Notaro,R., Luzzatto,L., Hill,A., Kelly,R., Hillmen,P., 2010. Paroxysmal nocturnal hemoglobinuria--hemolysis before and after eculizumab. N. Engl. J. Med. 363, 2270-2272.

68. Risitano,A.M., Notaro,R., Marando,L., Serio,B., Ranaldi,D., Seneca,E., Ricci,P., Alfinito,F., Camera,A., Gianfaldoni,G., Amendola,A., Boschetti,C., Di,B.E., Fratellanza,G., Barbano,F., Rodeghiero,F., Zanella,A., Iori,A.P., Selleri,C., Luzzatto,L., Rotoli,B., 2009. Complement fraction 3 binding on erythrocytes as additional mechanism of disease in paroxysmal nocturnal hemoglobinuria patients treated by eculizumab. Blood 113, 4094-4100.

69. Risitano,A.M., Notaro,R., Pascariello,C., Sica,M., Del,V.L., Horvath,C.J., Fridkis-Hareli,M., Selleri,C., Lindorfer,M.A., Taylor,R.P., Luzzatto,L., Holers,V.M., 2012. The complement receptor 2/factor H fusion protein TT30 protects paroxysmal nocturnal hemoglobinuria erythrocytes from complement-mediated hemolysis and C3 fragment. Blood 119, 6307-6316.

70. Risitano,A.M., Ricklin,D., Huang,Y., Reis,E.S., Chen,H., Ricci,P., Lin,Z., Pascariello,C., Raia,M., Sica,M., Del,V.L., Pane,F., Lupu,F., Notaro,R., Resuello,R.R., Deangelis,R.A., Lambris,J.D., 2014. Peptide inhibitors of C3 activation as a novel strategy of complement inhibition for the treatment of paroxysmal nocturnal hemoglobinuria. Blood. 123, 2094-101.

71. Risitano,A.M., Storek,M., Sahelijo,L., Doyle,M., Dai,Y., Weitz,I.C., Marsh,J.C.W., Elebute,M.O., O'Connell,C.L., Kulasekararaj,A.G., Ramsingh,G., Marotta,S., Hellmann,A., Lundberg,A.S. Safety and Pharmacokinetics of the Complement Inhibitor TT30 in a Phase I Trial for Untreated PNH Patients. ASH 57th Annual Meeting \& Exposition. 2015. Orlando, FL2015.

72. Rondelli,T., Risitano,A.M., Peffault de,L.R., Sica,M., Peruzzi,B., Ricci,P., Barcellini,W., lori,A.P., Boschetti,C., Valle,V., Fremeaux-Bacchi,V., De,A.M., Socie,G., Luzzatto,L., Notaro,R., 2014. Polymorphism of the complement receptor 1 gene correlates with the hematologic response to eculizumab in patients with paroxysmal nocturnal hemoglobinuria. Haematologica 99, 262-266. 
73. Rother,R.P., Rollins,S.A., Mojcik,C.F., Brodsky,R.A., Bell,L., 2007. Discovery and development of the complement inhibitor eculizumab for the treatment of paroxysmal nocturnal hemoglobinuria. Nat. Biotechnol. 25, 1256-1264.

74. Roversi,P., Lissina,O., Johnson,S., Ahmat,N., Paesen,G.C., Ploss,K., Boland,W., Nunn,M.A., Lea,S.M., 2007. The structure of $\mathrm{OMCl}$, a novel lipocalin inhibitor of the complement system. J. Mol. Biol. 369, 784-793.

75. Sacks,S.H., Zhou,W., 2012. The role of complement in the early immune response to transplantation. Nat. Rev. Immunol. 12, 431-442.

76. Sahu,A., Kay,B.K., Lambris,J.D., 1996. Inhibition of human complement by a C3-binding peptide isolated from a phage-displayed random peptide library. J. Immunol. 157, 884-891.

77. Sahu,A., Morikis,D., Lambris,J.D., 2003. Compstatin, a peptide inhibitor of complement, exhibits speciesspecific binding to complement component C3. Mol. Immunol. 39, 557-566.

78. Sahu,A., Soulika,A.M., Morikis,D., Spruce,L., Moore,W.T., Lambris,J.D., 2000. Binding kinetics, structureactivity relationship, and biotransformation of the complement inhibitor compstatin. J. Immunol. 165, 2491-2499.

79. Santoro,D., Satta,E., Messina,S., Costantino,G., Savica,V., Bellinghieri,G., 2013. Pain in end-stage renal disease: a frequent and neglected clinical problem. Clin. Nephrol. 79 Suppl 1, S2-11.

80. Schmidt,C.Q., Bai,H., Lin,Z., Risitano,A.M., Barlow,P.N., Ricklin,D., Lambris,J.D., 2013. Rational engineering of a minimized immune inhibitor with unique triple-targeting properties. J. Immunol. 190, 5712-5721.

81. Schmidt,C.Q., Harder,M.J., Nichols,E.M., Hebecker,M., Anliker,M., Hochsmann,B., Simmet,T., Csincsi,A.I., Uzonyi,B., Pappworth,I.Y., Ricklin,D., Lambris,J.D., Schrezenmeier,H., Jozsi,M., Marchbank,K.J., 2016. Selectivity of C3-opsonin targeted complement inhibitors: A distinct advantage in the protection of erythrocytes from paroxysmal nocturnal hemoglobinuria patients. Immunobiology 221, 503-511.

82. Schramm,E.C., Clark,S.J., Triebwasser,M.P., Raychaudhuri,S., Seddon,J.M., Atkinson,J.P., 2014. Genetic variants in the complement system predisposing to age-related macular degeneration: a review. Mol. Immunol. 61, 118-125.

83. Servais,A., Noel,L.H., Roumenina,L.T., Le,Q.M., Ngo,S., Dragon-Durey,M.A., Macher,M.A., Zuber,J., Karras,A., Provot,F., Moulin,B., Grunfeld,J.P., Niaudet,P., Lesavre,P., Fremeaux-Bacchi,V., 2012. Acquired and genetic complement abnormalities play a critical role in dense deposit disease and other C3 glomerulopathies. Kidney Int. 82, 454-464.

84. Sethi,S., Fervenza,F.C., 2014. Pathology of renal diseases associated with dysfunction of the alternative pathway of complement: C3 glomerulopathy and atypical hemolytic uremic syndrome (aHUS). Semin. Thromb. Hemost. 40, 416-421.

85. Silasi-Mansat,R., Zhu,H., Popescu,N.I., Peer,G., Sfyroera,G., Magotti,P., Ivanciu,L., Lupu,C., Mollnes,T.E., Taylor,F.B., Kinasewitz,G., Lambris,J.D., Lupu,F., 2010. Complement inhibition decreases the procoagulant 
response and confers organ protection in a baboon model of Escherichia coli sepsis. Blood 116, $1002-$ 1010.

86. Smith,R.J., Alexander,J., Barlow,P.N., Botto,M., Cassavant,T.L., Cook,H.T., de Cordoba,S.R., Hageman,G.S., Jokiranta,T.S., Kimberling,W.J., Lambris,J.D., Lanning,L.D., Levidiotis,V., Licht,C., Lutz,H.U., Meri,S., Pickering,M.C., Quigg,R.J., Rops,A.L., Salant,D.J., Sethi,S., Thurman,J.M., Tully,H.F., Tully,S.P., van,d., V, Walker,P.D., Wurzner,R., Zipfel,P.F., 2007. New approaches to the treatment of dense deposit disease. J. Am. Soc. Nephrol. 18, 2447-2456.

87. Sprong T1, Brandtzaeg P, Fung M, Pharo AM, Høiby EA, Michaelsen TE, Aase A, van der Meer JW, van Deuren M, Mollnes TE., 2003. Inhibition of C5a-induced inflammation with preserved C5b-9-mediated bactericidal activity in a human whole blood model of meningococcal sepsis Blood. 102, 3702-10.

88. Stegall,M.D., Chedid,M.F., Cornell,L.D., 2012. The role of complement in antibody-mediated rejection in kidney transplantation. Nat. Rev. Nephrol. 8, 670-678.

89. Swedish Orphan Biovitrum, 2016. Safety and Tolerability of SOBIO02 in Healthy Volunteers Following Single and Repeated Administration. https://clinicaltrials.gov/ct2/show/NCT02083666?term=SOBI\&rank=1.

90. Takeda,J., Miyata,T., Kawagoe,K., lida,Y., Endo,Y., Fujita,T., Takahashi,M., Kitani,T., Kinoshita,T., 1993. Deficiency of the GPI anchor caused by a somatic mutation of the PIG-A gene in paroxysmal nocturnal hemoglobinuria. Cell 73, 703-711.

91. Teramura,Y., Asif,S., Ekdahl,K.N., Nilsson,B., 2015. Cell Surface Engineering for Regulation of Immune Reactions in Cell Therapy. Adv. Exp. Med. Biol. 865, 189-209.

92. Wang,J., Wang,L., Xiang,Y., Ricklin,D., Lambris,J.D., Chen,G., 2016. Using an in vitro xenoantibodymediated complement-dependent cytotoxicity model to evaluate the complement inhibitory activity of the peptidic C3 inhibitor Cp40. Clin. Immunol. 162, 37-44.

93. Woodruff,T.M., Nandakumar,K.S., Tedesco,F., 2011. Inhibiting the C5-C5a receptor axis. Mol. Immunol. 48, 1631-1642.

94. Wu MC, Brennan FH, Lynch JP, Mantovani S, Phipps S, Wetsel RA, Ruitenberg MJ, Taylor SM, Woodruff TM., 2013. The receptor for complement component C3a mediates protection from intestinal ischemiareperfusion injuries by inhibiting neutrophil mobilization. Proc Natl Acad Sci U S A. 110, 9439-44.

95. Wu,Y.Q., Qu,H., Sfyroera,G., Tzekou,A., Kay,B.K., Nilsson,B., Nilsson,E.K., Ricklin,D., Lambris,J.D., 2011. Protection of nonself surfaces from complement attack by factor $\mathrm{H}$-binding peptides: implications for therapeutic medicine. J. Immunol. 186, 4269-4277.

96. Xiao,X., Pickering,M.C., Smith,R.J., 2014. C3 glomerulopathy: the genetic and clinical findings in dense deposit disease and C3 glomerulonephritis. Semin. Thromb. Hemost. 40, 465-471. 
97. Zhang,Y., Nester,C.M., Holanda,D.G., Marsh,H.C., Hammond,R.A., Thomas,L.J., Meyer,N.C., Hunsicker,L.G., Sethi,S., Smith,R.J., 2013. Soluble CR1 therapy improves complement regulation in C3 glomerulopathy. J. Am. Soc. Nephrol. 24, 1820-1829.

98. Zhang,Y., Shao,D., Ricklin,D., Hilkin,B.M., Nester,C.M., Lambris,J.D., Smith,R.J., 2015. Compstatin analog Cp40 inhibits complement dysregulation in vitro in C3 glomerulopathy. Immunobiology 220, 993-998. 


\section{Figure Legend}

Figure 1. (Panel A) A simplified schematic overview of the complement cascade with points of therapeutic intervention by clinically developed C3 inhibitors. Complement activation on foreign surfaces (e.g. microbial, transplants, biomaterials) or damaged/transformed self surfaces can be triggered by any of the three pathways (classical, lectin, alternative). All pathways converge at the cleavage of the central component, $\mathrm{C} 3$, by the C3 convertases. Amplification of the AP is considered a pivotal pathogenic driver in many complement-mediated diseases. Activation of C5 by C5 convertases of both the classical and alternative pathway leads to the release of the proinflammatory mediator C5a and the assembly of the cell-perforating MAC (C5b-9). For simplicity reasons only the alternative

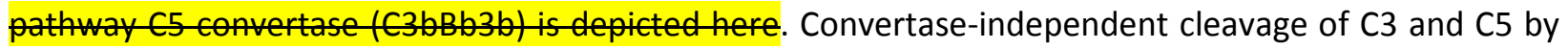
coagulation and fibrinolytic proteases (e.g. plasmin, thrombin) has been implicated as a bypass pathway of complement activation in certain pathophysiological contexts. C3-targeted inhibitors (e.g. Cp40, AMY-101) can provide effective and broad therapeutic control of the cascade by targeting all pathways at the level of C3 and blocking downstream complement effector responses (e.g. C5a generation, MAC assembly). Abbreviations: AP, alternative pathway; MBL, mannose-binding lectin; MASPs, mannosebinding lectin associated proteases; FP, properdin; FB, factor B; FD factor D; MAC, membrane attack complex.

(Panel B) Concepts and illustrative examples of C3-targeted therapeutic modulation. Several inhibitory approaches have been developed and are currently in clinical evaluation as C3-targeted therapeutics for various indications. These include: a) systemic or local complement modulation at the level of C3 (e.g. compstatin analogs), b) surface-targeting of fluid-phase complement regulators ( e.g. FH) acting at the level of the AP C3 convertase (e.g. mini-FH variants, CR2-FH fusion constructs:TT30) and c) targeting moieties that recruit endogenous C3 regulators to the complement-opsonized surface (e.g. FH-binding peptides, 5C6). Blue arrows indicate ways by which AP C3 convertase activity can be regulated by natural, chimeric or small-sized C3 inhibitors. 


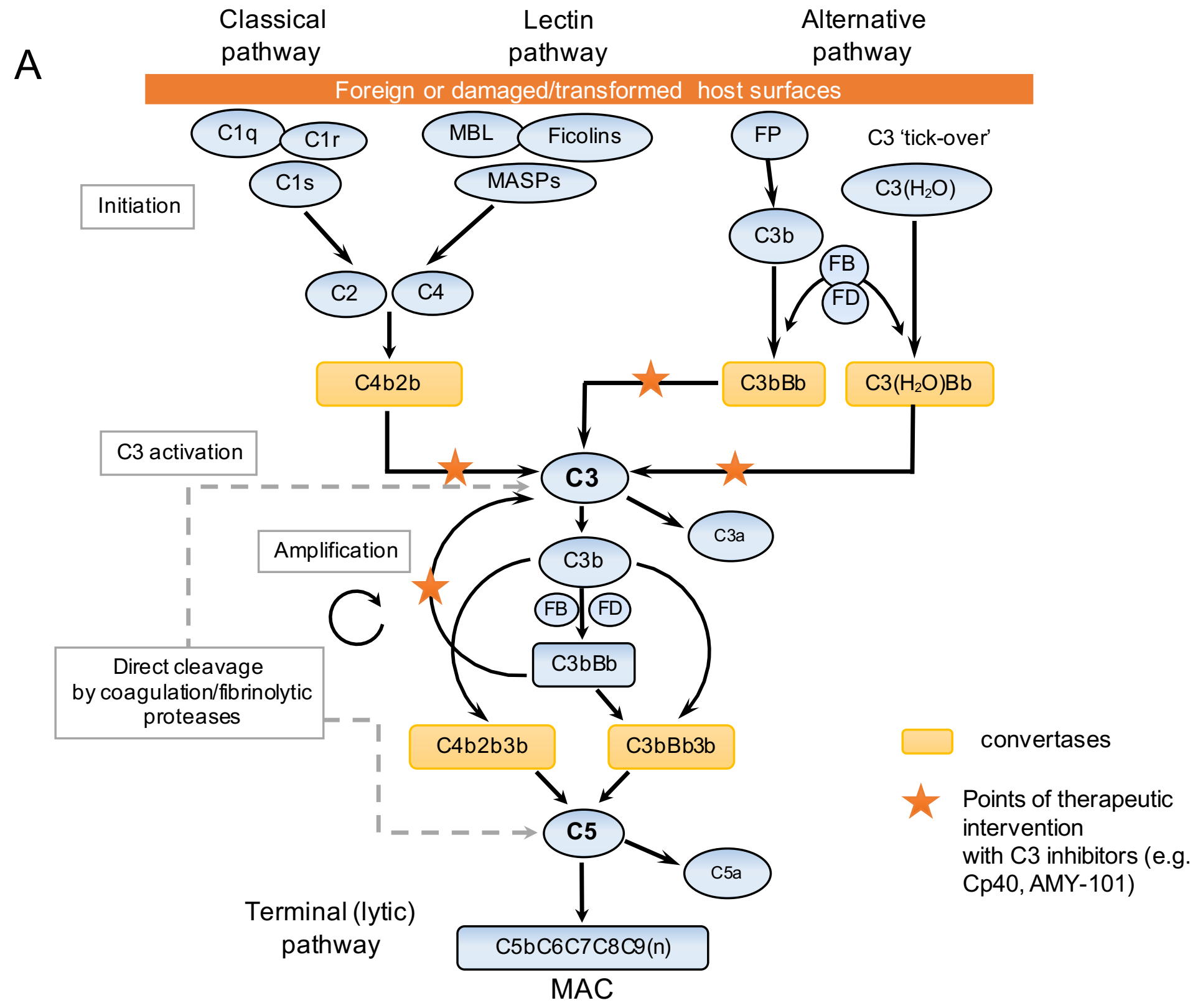

\section{B Strategies for therapeutic modulation of the AP C3 convertase}

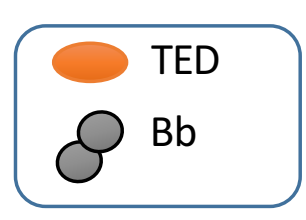

Compstatins

(e.g. Cp40, AMY-101)
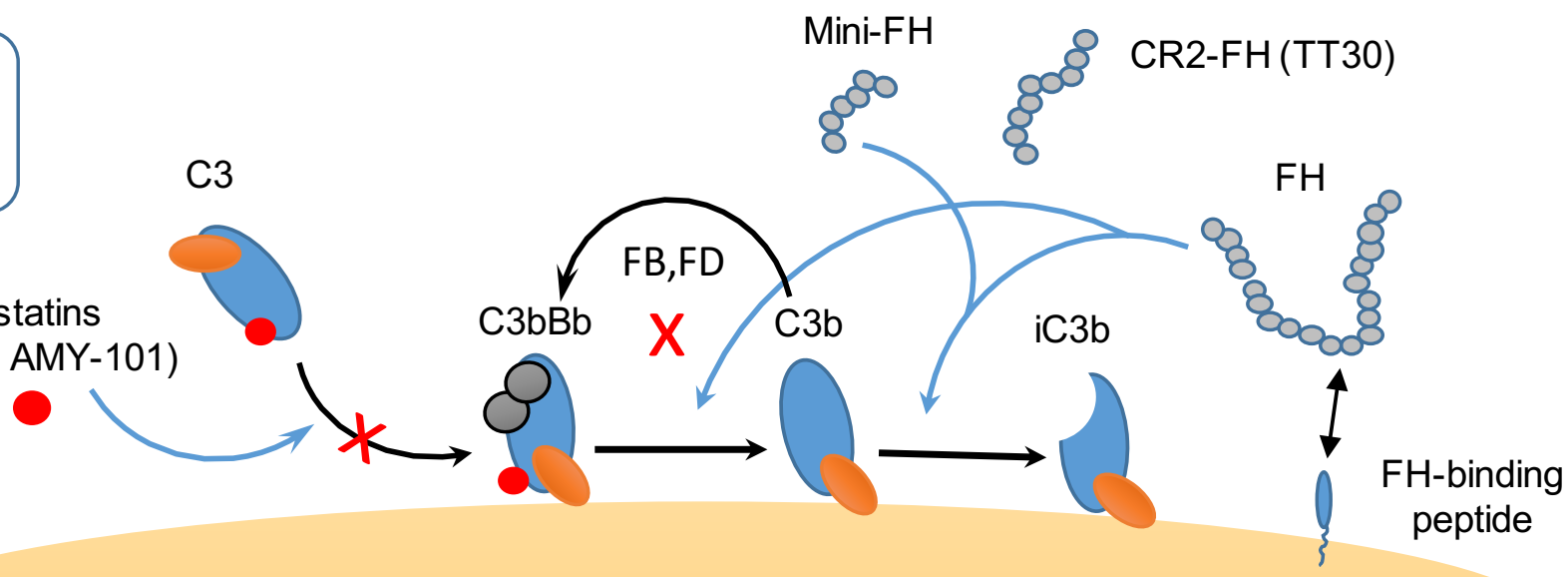

Complement-targeted surface 